\title{
THE APPLICATION OF A \\ HHT BASED ULTRASONIC DETECTING METHOD IN QUALITY ASSESSMENT OF CFST
}

\author{
Fu-xiang $\mathrm{Ye}^{1}$, Xiao-xiong $\mathrm{Zha}^{2, *}$ and Hong-xin $\mathrm{Wang}^{3}$ \\ ${ }^{1}$ Shenzhen Graduate School, Harbin Institute of Technology, Shenzhen 518055, China \\ ${ }^{2}$ Professor, Department of Urban and Civil Engineering, Shenzhen Graduate School, \\ Harbin Institute of Technology, China \\ ${ }^{3} \mathrm{PhD}$ Candidate, Shenzhen Graduate School, Harbin Institute of Technology, Shenzhen 518055, China \\ *(Corresponding author: E-mail: zhaxx@hit.edu.cn)
}

Received: 14 October 2010; Revised: 21 November 2010; Accepted: 26 November 2010

\begin{abstract}
Owed to the features of concrete and concrete pumping construction craft, empty holes, osteoporosis, construction joints and poor cementation often appear inside CFST. In view of this situation, ultrasonic detecting method has been developed to assess the quality of CFST. However, traditional methods are limited because of the effect of noise signal and distortion of signal. In this paper, the filtering process and HHT were applied to the ultrasonic signal received, and the Hilbert energy spectrum and the first-order intrinsic mode function were used to assess the quality of CFST. Actual testing results show that the HHT analysis method, combined with filtering process, can effectively improve the identification of the arrival time, the amplitude value, the waveform and the frequency of the first wave of the ultrasonic signal, and thus improve the damage identification quality of CFST.
\end{abstract}

Keywords: Hilbert-Huang transform; hilbert energy spectrum; ultrasonic detecting; bandpass digital filter; CFST

\section{INTRODUCTION}

The concrete-filled steel tube (CFST) is a kind of bearing component made by pumping concrete into a prefabricated steel tube. Advantages of this structure such as high bearing capacity, good ductility, good aseismic performance, easy constructing, short constructing period, thus CFST structures have been widely used in all kinds of high-rise buildings, heavy-duty workshops and highway bridge projects. However, affected by the features of concrete and the concrete pumping construction craft, empty holes, osteoporosis, construction joints and poor cementations often appear inside CFST, which may directly influence the quality of CFST. Therefore, it is necessary to assess construction quality of CFST adopt scientific and effective method [1].

Hilbert-Huang Transform (HHT) has been considered as a significant breakthrough in recent years to the linear and steady-state spectral analysis which is based on the Fourier transform, especially for it can show the time-frequency information of the signal. This method adopts the multi-scale analysis advantage of the wavelet analysis, meanwhile, overcome the difficulty of the wavelet analysis in needing to select the suitable wavelet basis. Moreover, it can analyze not only linear stationary signals, but also the linear non-stationary signals by self-adapting. In this paper, the HHT is applied to the experiment investigation by using the ultrasonic wave to detect the delamination, sundry and empty hole defects in CFST, the Hilbert energy spectrum and the first-order intrinsic mode functions of the ultrasonic wave signals is used to assess the quality of CFST. It shows that the HHT analysis method, combined with filtering process, can effectively improve the identification of the arrival time, the amplitude value, the waveform and the frequency of the first wave of the ultrasonic signal, which can improve the damage identification of CFST quality. 


\section{EXPERIMENTAL SPECIMEN}

The experimental specimen is a CFST column, the height of which is $700 \mathrm{~mm}$, outer diameter is 219 $\mathrm{mm}$ and thickness of steel tube is $6 \mathrm{~mm}$. Before the experiments, three kinds of defects of specimen are prefabricated made as follow: 1) At the bottom of the column, a thin timber packed in the plastic membrane is used to separate out a hole to create the delamination defect, and after the initial solidification of concrete the timber is uprooted in Figure 1;2) In the middle of the CFST column, a timber block is vertically embedded into the concrete to create the sundry defect. As shown in Figure 2, the dimension of the timber block is $150 \mathrm{~mm} \times 50 \mathrm{~mm} \times 25 \mathrm{~mm}$; 3) As shown in Figure 3, for creating the hole defect, a $200 \mathrm{~mm}$-long cylinder hole is made with diameter gradually increasing from $10 \mathrm{~mm}$ to $20 \mathrm{~mm}$, at the center of the section, in the upper part of the CFST column.

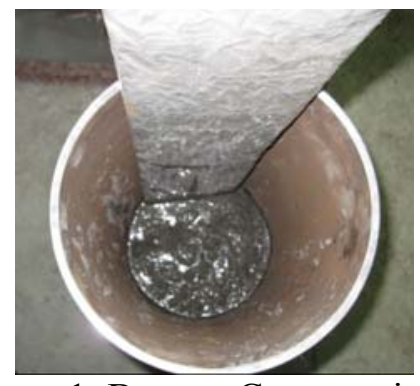

Figure 1. Bottom Construction

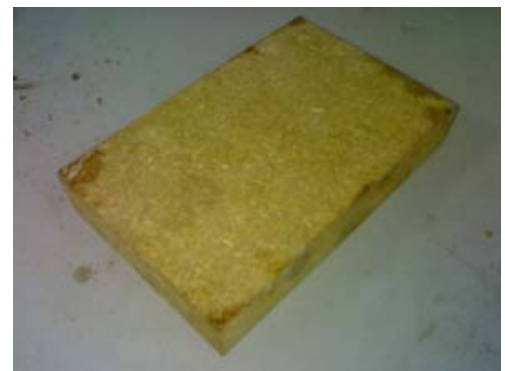

Figure 2. The Timber Block

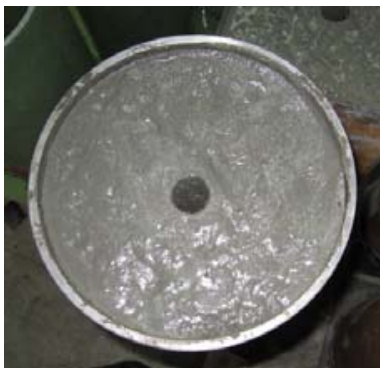

Figure 3. The Hole in the Upper Part

\section{TEST METHOD}

\subsection{Prerequisites of the Test Method}

As it is shown in Figure 4(a), in CFST with good quality, the signal expected to be received is the signal which penetrate the steel tube along the radial direction of steel tube, then propagate through the concrete in the steel tube and then penetrate the steel tube again to reach the receiver; and signal propagate along the half circumference of steel tube to reach the receiver is considered as interfering signal.

The ultrasonic time measured by the receiver is the arrival time of the first ultrasonic wave, if the ultrasonic propagation along the half circumference of steel tube arrive earlier than the ultrasonic propagation through the core concrete, the ultrasonic wave method to measure the quality of concrete-filled steel tube would be meaningless.

Denote the time the ultrasonic propagate along the radial direction of the CFST as $t_{1}$, the time the ultrasonic propagate along the half circumference of steel tube as $t_{2}$, then the following relationship between $t_{1}$ and $t_{2}$ is shown in Eq. 1 (Due to the thickness of steel tube is far less than the diameter of the core concrete, the penetration time of the ultrasonic through the steel tube is ignored.):

$\frac{t_{2}}{t_{1}}=\frac{\pi}{2} \cdot \frac{V_{1}}{V_{2}}$

Where: $V_{1}, V_{2}$ are velocities of the ultrasonic in the concrete and in the steel tube, respectively $(\mathrm{m} / \mathrm{s})$. 


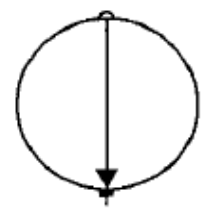

(a)

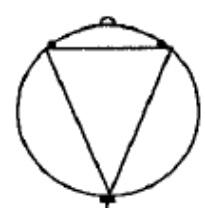

(b)

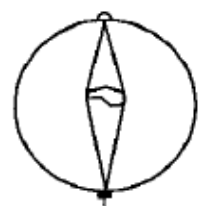

(c)

Figure 4. Paths of Ultrasonic in CFST

Ultrasonic velocity in Steel tube is about $5700 \mathrm{~m} / \mathrm{s}$, therefore, to satisfy the request of $t_{2} / t_{1}>1$, the ultrasonic velocity in concrete must greater than $3628 \mathrm{~m} / \mathrm{s}$. And the label of the concrete usually used in CFST is C30, whose ultrasonic velocity is about $4400 \mathrm{~m} / \mathrm{s}$. Obviously, when the concrete and steel tube is close-grained and of good cementation, the first wave received in penetrating detecting method is the ultrasonic wave propagate along the radial direction of CFST, and the ultrasonic wave propagate along the half circumference of steel tube arrived the receiver later. Thus, the application of ultrasonic testing method to the CFST quality is feasible and efficient.

When the concrete and steel tube is in poor cementation condition or even delaminated, the ultrasonic wave propagating path in concrete is as show in Figure 4(b), when there is a hole in the concrete the ultrasonic wave propagating path in concrete is as show in Figure 4(c). In these two conditions, time needed for the ultrasonic wave to arrive the receiver is longer than time needed in the compact concrete, expressing as the ultrasonic velocity in concrete with defects is smaller. Thus, the method of inspecting the arrival time of the first ultrasonic wave is practicable in detecting defects of CFST.

In addition, the amplitude, the distortion degree and the frequency of the first wave signal can also be used to judge the casting quality inside CFST $[2,3]$.

\subsection{Hilbert-Huang Transform}

The Hilbert-Huang transform is composed of two parts: Empirical mode decomposition (EMD) and Hilbert transform (HT). The key point of this method is the Empirical mode decomposition; it can process linearization and smoothing process on nonlinear, non-stationary signals, keeping the characteristic of the signal $[4,5]$. Any complicated data can be directly extracted energy in proper time range, which would generate a group of intrinsic mode functions who have very good Hilbert transformation properties. Then, after be applied the Hilbert transform, the intrinsic mode functions (IMF) would possess instantaneous frequency and instantaneous amplitude, the distribution of the instantaneous amplitude in the frequency-time plane is called the Hilbert spectrum, indicating the relationship among energy, frequency and time [6,7], the Hilbert energy spectrum can be obtained by integrating the square of the amplitude in the time domain.

\subsubsection{Empirical mode decomposition method (EMD)}

The basic thought of the Empirical mode decomposition method is to use the average value of the upper and lower envelopes of the signal to determine "the instantaneous average balance position", and then extract the intrinsic mode functions. The upper and the lower envelope of the signal are obtained by fitting the maximum points and the minimum points by cubic-spline interpolation method, respectively. 
The basic process of EMD process can be summarized as follows:

(1) Find out all local maximas and local minimums of signal $x(t)$. In order to retain the characteristics of the original signal better, local maxima is defined as a value in a moment of the time series, who meet the condition that is greater than both the value of just a moment before and the value of just a moment after. The local minimum value is defined similarly. Then, using cubic-spline function to fit the data, the upper envelope $x_{\max }(t)$ and the lower envelope $x_{\min }(t)$ of the signal are got;

(2) Calculate the mean value of the upper envelope and the lower envelope: $m(t)=\left[x_{\max }(t)+x_{\min }(t)\right] / 2$;

(3) Subtracting the original signal $x(t)$ by the mean value $m(t)$, the first component $h(t)=x(t)-m(t)$ can be got. Due to the differences between original sequences, component $h(t)$ may not be an IMF. If $h(t)$ does not meet the two conditions of IMF: Within the data scope, the difference of the number of extreme value points and the times of the data sequence across the zero is less than two; At any point, the mean value of the envelope formed by all the maximum points and the envelope formed by all the minimum points is equal to zero. Then treat $h(t)$ as the original signal, and repeat processes (1) - (3), until the two conditions are met. Then $h(t)$ can be treated as an IMF, let $I_{1}(t)=h(t)$, till now the first IMF has been successfully extracted. Due to the fact that: the rest of the signal $r_{1}(t)=x(t)-I_{1}(t)$ still contains information with longer periodic components, so it can be treated as a new signal, and repeat the extracting process. Similarly, $I_{2}(t), I_{3}(t), \ldots I_{n}(t)$ can be got. When $r_{n}(t)$ is a drab sequence or a constant sequence, extracting processes would be terminated, the task of extracting IMFs is completed, and $r_{n}(t)$ is called the over trend term of the original signal. So the signal $x(t)$ can be expressed as $x(t)=\sum_{i=1}^{n} I_{i}(t)+r_{n}(t)$, that is the original signal is composed of $n$ IMFs and an over trend term. As it is mentioned above, the whole process is just like a selecting process, to extract the IMFs from the signal according to the time characteristics.

\subsubsection{Hilbert transform}

For stochastic time sequence $X(t)$, applying Hilbert transform to it [8],Y(t) can be got as follow Eq. 2 :

$Y(t)=\frac{1}{\pi} P V\left(\int_{-\infty}^{\infty} \frac{X(\tau)}{t-\tau} d \tau\right)$

Where, $P V$ is the Cauchy principal value. This equation indicate that $Y(t)$ is the convolution of $X(t)$ and $\frac{1}{\pi \tau}$. Through this definition, $X(t)$ and $Y(t)$ formed a conjugated-complex-number couple, then an analytical signal $Z(t)$ can be defined as Eq. 3 :

$Z(t)=X(t)+i Y(t)=a(t) e^{i \theta(t)}$ 
Where, $a(t)$ and $\theta(t)$ can be defined as Eq. 4 :

$$
\left\{\begin{array}{l}
a(t)=\left[X^{2}(t)+Y^{2}(t)\right]^{1 / 2} \\
\theta(t)=\arctan \left(\frac{Y(t)}{X(t)}\right)
\end{array}\right.
$$

As the phase of the signal is got, because the instantaneous frequency is the derivative of the phase, the instantaneous frequency can be expressed as Eq. 5 :

$$
w=\frac{d \theta(t)}{d t}
$$

After applied Hilbert transform to intrinsic mode functions, the signal can be expressed Eq. 6 :

$$
X(t)=\sum_{i=1}^{n} a_{j}(t) \exp \left(i \int w_{i}(t) d t\right)+r_{n}(t)
$$

Due to the energy in signal $r_{n}(t)$ is very small, it is ignored here.

The amplitude and the frequency obtained from Hilbert transform are both function of time, either using 3D graphic to express the relationship among amplitude, frequency and time, or displaying the amplitude with gray form graphic in the frequency-time plane, the Hilbert spectrum $H(w, t)$ can both be got. Integrating the square of the amplitude of energy in the time domain, the Hilbert energy spectrum is got according to Eq. 7 :

$$
E S(w)=\int_{T} H^{2}(w, t) d t
$$

The Hilbert energy spectrum provides the energy measurement to each frequency, and expresses the energy accumulation of each frequency in the whole length of time.

\subsection{Preprocessing to Ultrasonic Signal}

The original signal emitted from the Ultrasonic detector is a narrowband signal with a center frequency $45 \mathrm{KHz}$, and the signal received by the receiver has induced the low-frequency and high-frequency noise signal. Based on the matlab software, the Kaiser-window bandpass digital filter is used to filter out the noise signal in the ultrasonic signals, setting the lower frequency as $10 \mathrm{KHz}$ and the upper frequency as $80 \mathrm{KHz}$. Considering that during the filtering process, an overall phase delay would be induced into the signal, which would also leads to a loss of the data and information at the end of the original signal. In order to eliminate this loss and delay, before the filtering process, zeros are added to the end of the signal, the length of zeros is set to be the length of the phase delay time; and after the filtering process, data with the same length are removed from the front part of the signal, and finally the graphs of the signal before and after the filtering process (Figure 5 and Figure 6) and their corresponding Hilbert energy spectrum graphs (Figure 7 and Figure 8) are got. It can be found that: after the filtering process, the signal has become noticeably smooth, with no burr, greatly convenient for the identification of the first wave time. Meanwhile, by comparing the Hilbert energy spectrums before and after the filtering process, it can be found that the bandpass digital filter does a good job in filtering out the low-frequency noise signal. 


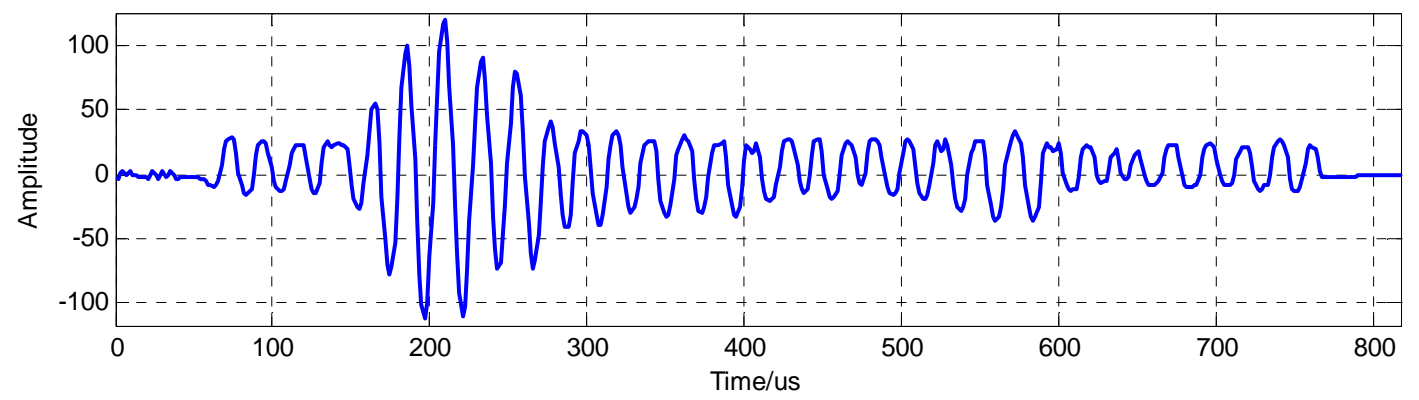

Figure 5. Signal before Filtering Process

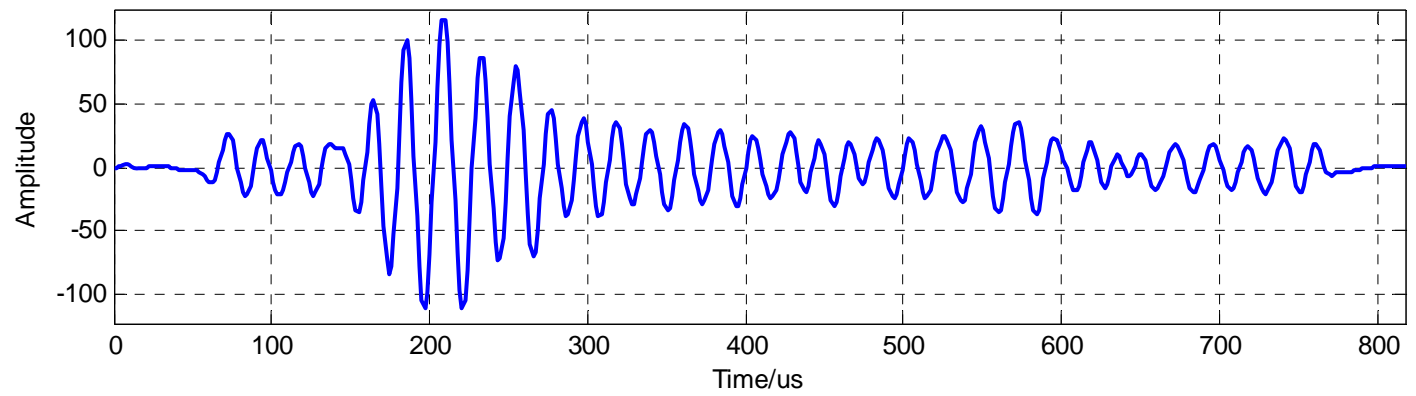

Figure 6. Signal after Filtering Process

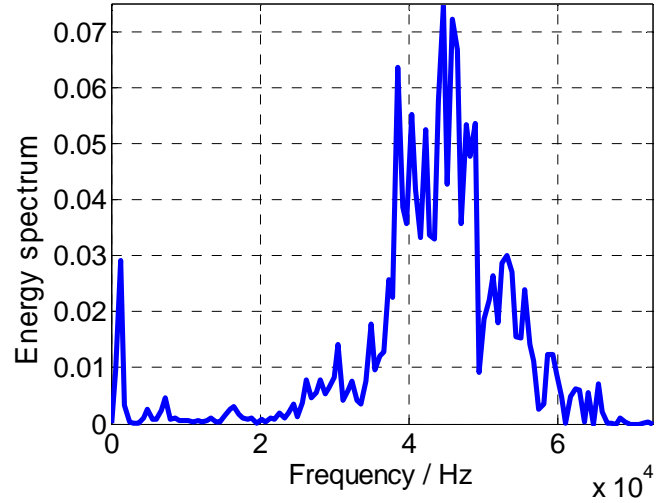

Figure 7. Hilbert Energy Spectrums before Filtering Process

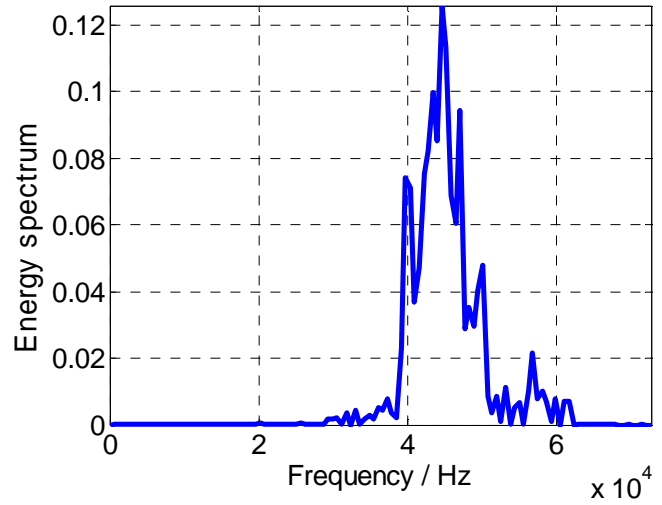

Figure 8. Hilbert Energy Spectrums after Filtering Process

\section{EXPERIMENT RESULT}

In order to verify the effectiveness of applying the HHT into the ultrasonic detection technology of the quality inspection of CFST, ultrasonic detection technology is applied to the experimental model mentioned above, and after the filtering process, the ultrasonic signal is dealt with HHT. Comparing the Hilbert energy spectrums and the first-order intrinsic mode functions, the results are obtained as follows.

As the diameter of the steel tube is $219 \mathrm{~mm}$ and the ultrasonic velocity in concrete is $4400 \mathrm{~m} / \mathrm{s}$, the ultrasonic velocity in the steel tube propagating in the form of surface wave is $2900 \mathrm{~m} / \mathrm{s}$, time for the ultrasonic to propagate through the core concrete to the receiver can be calculated by: $t_{1}=0.219 \div 4400=49.8 \mu \mathrm{s}$, and time for the ultrasonic to propagate along the half circumference of steel tube to reach the receiver can be calculated by: $t_{2}=0.219 \times \pi \div 2 \div 2900=118.6 \mu$ s . 


\subsection{Comparison of Undamaged Position and Delaminated Position}

Comparing Figure 9 and Figure 10, it can be found that: Attached with filtering process, the intrinsic mode decomposition results can effectively suppress the noise signals. Analysis results show that: For no defect position, much energy of ultrasonic propagate directly through the core concrete and reach the receiver, obvious waveform can be observed from $50 \mu \mathrm{s}$; In the position with delaminating defect, only a few energy propagate directly through the air into the core concrete, and most energy propagate in the form of surface wave along the half circumference of steel tube to reach the receiver, thus till around $120 \mu \mathrm{s}$, obvious waveform can be observed.

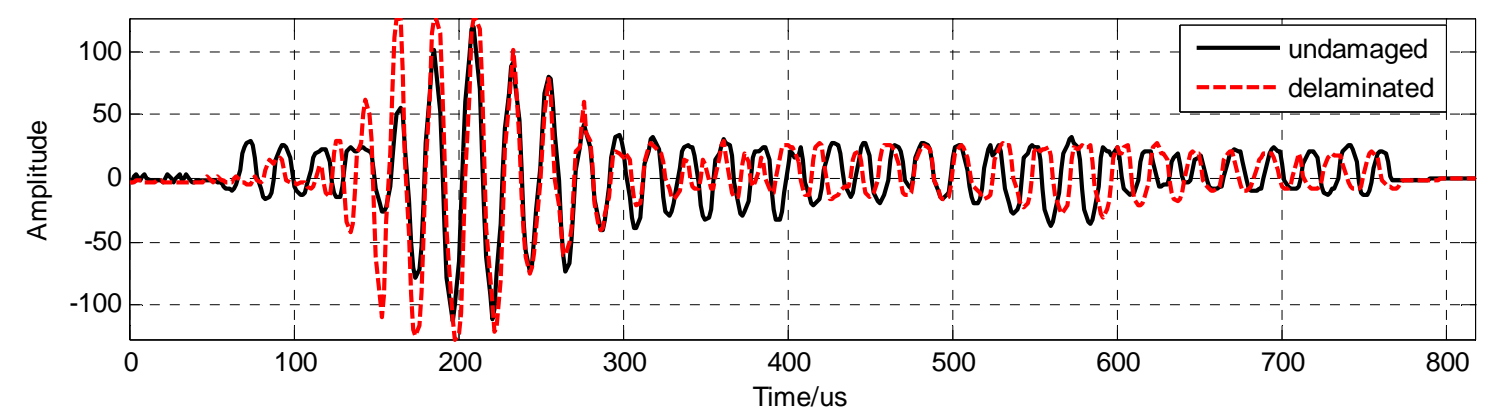

Figure 9. Waveform Comparison before Filtering

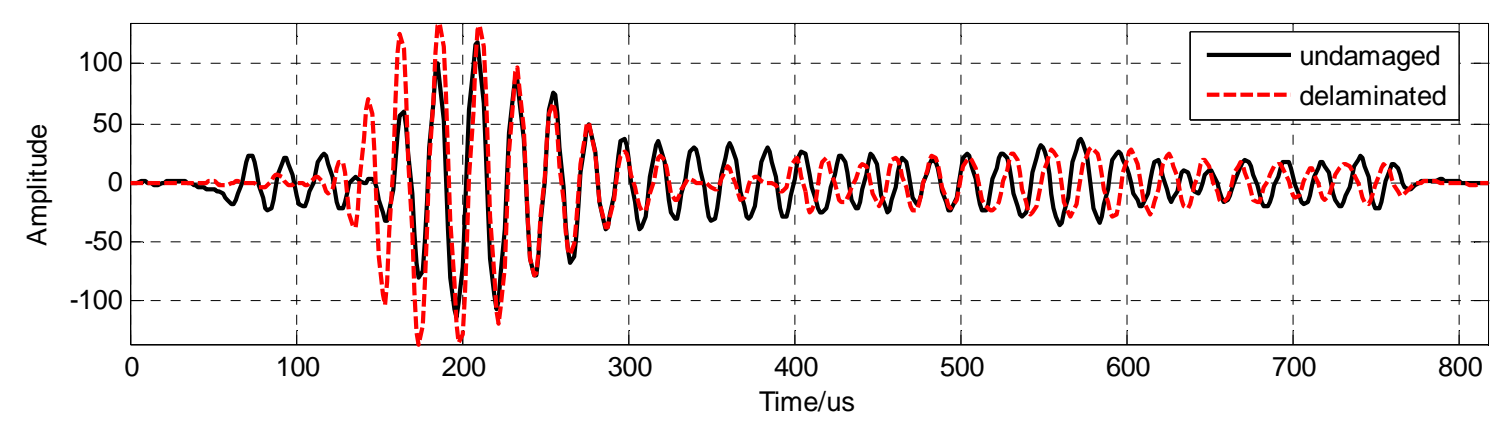

Figure 10. Comparison of the First-order Intrinsic Mode Functions of Undamaged Position and Delaminated Position

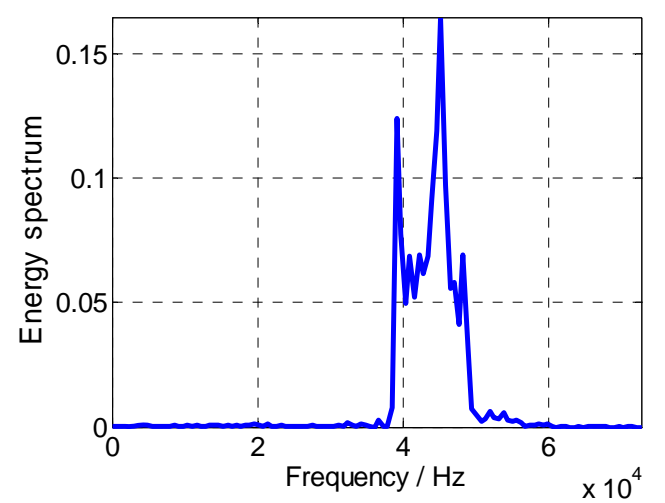

Figure 11. Energy Spectrum of Undamaged Position

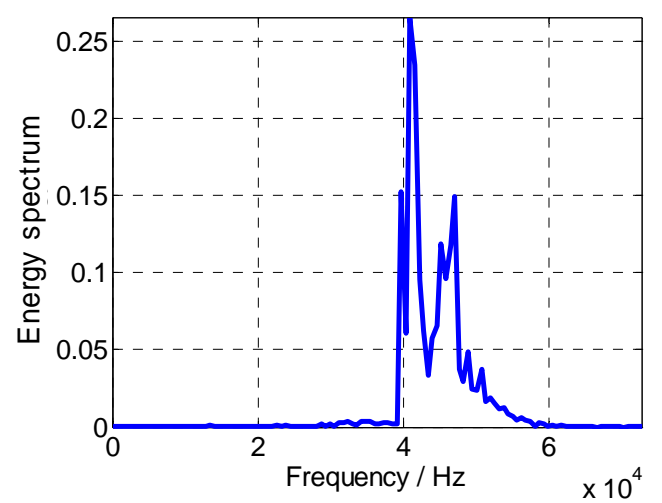

Figure 12. Energy Spectrum of Delaminated Position 
Comparing Figure 11 and Figure 12, it can be found that: Amplitude of energy spectrum in delaminated position is obviously higher than that in undamaged position, and energy in the high frequency domain in delaminated position is also greater than that in undamaged position. That is because: In delaminated position, most energy propagates in steel, resulting in less attenuation of energy.

\subsection{Comparison of Undamaged Position and Position with Sundry Inside}

Observing the two wave lines in Figure 13, conclusion can be made as follow: For undamaged position, obvious waveform can be observed from $50 \mu \mathrm{s}$; for position with sundry inside, obvious waveform cannot be observed until about $80 \mu$ s .

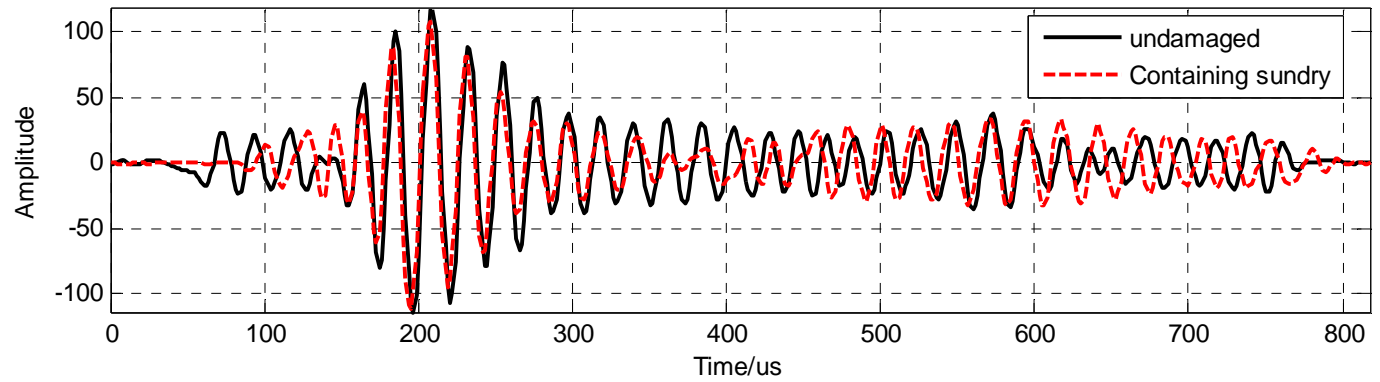

Figure 13. Comparison of the First-order Intrinsic Mode Functions of Undamaged Position and Position with Sundry Inside

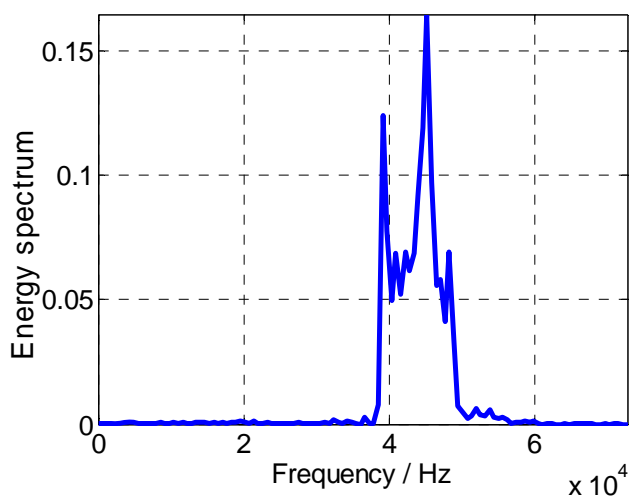

Figure 14. Energy Spectrum of Undamaged Position

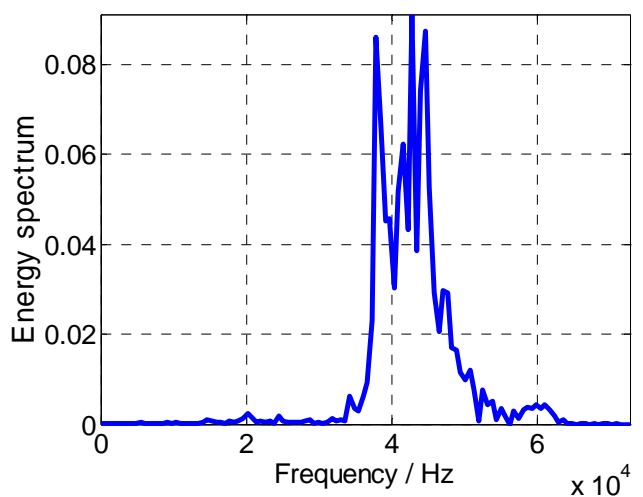

Figure 15. Energy Spectrum of Position with Sundry Inside

Inspecting Figure 14 and Figure 15, obvious difference can be found: the amplitude and the peak value of the energy spectrum in position with sundry inside is notably smaller than that in undamaged position. That is because some of the ultrasonic energy is lost due to the reflection on the boundary surface between the sundry and the concrete.

\subsection{Comparison of Undamaged Position and Position with Hole in the Section Center}

Detect position with a $1.5 \mathrm{~mm}$ diameter hole at the section center with ultrasonic signal and then compare the results of it with the results of undamaged position as follow.

As it is shown in Figure 15, the first wave arrival time of the first-order IMF of the position with hole is a little late than that of undamaged position. Moreover, the two curves in Figure 16 can be observed obvious phase difference throughout the sampling time, the curve of the position with hole always arrive the peak value before the curve of the undamaged position. 


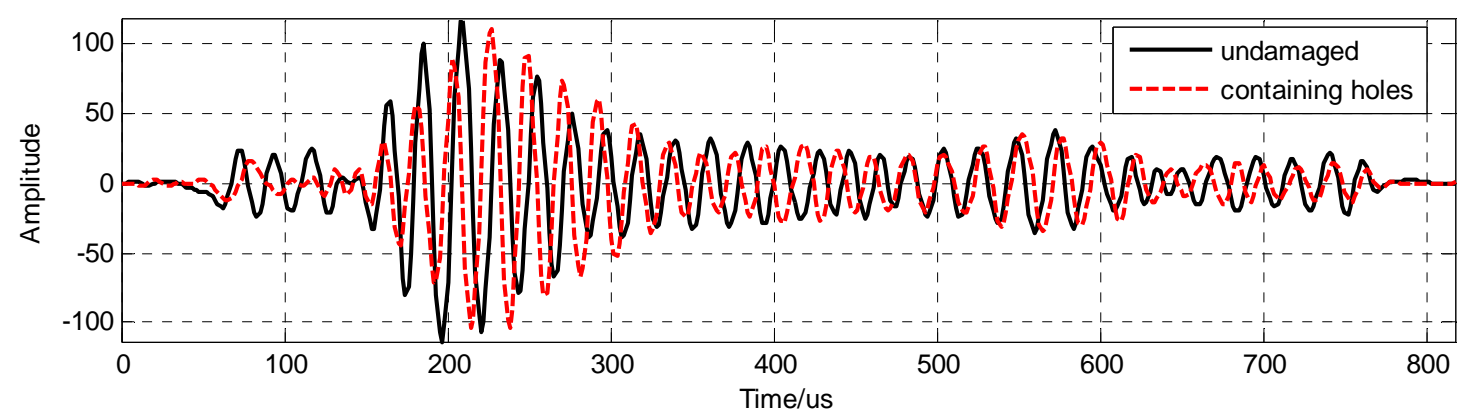

Figure 16. Comparison of the First-order Intrinsic Mode Functions of Undamaged Position and Position with Hole

Comparing Figure 17 with Figure 18, only very small difference can be observed, the amplitude of energy at about $40 \mathrm{KHz}$ in position with hole is smaller than that in undamaged position. It can be expected that when the diameter of the hole is very small, the effect of it to the energy spectrum may be hard to be identified.

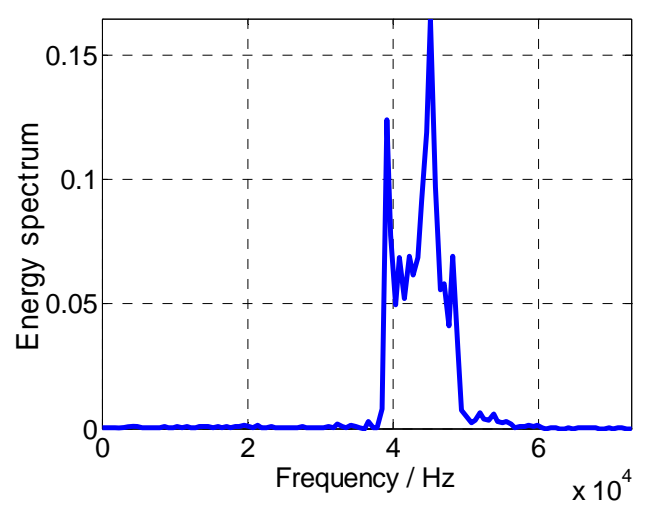

Figure 17. Energy Spectrum of Undamaged Position

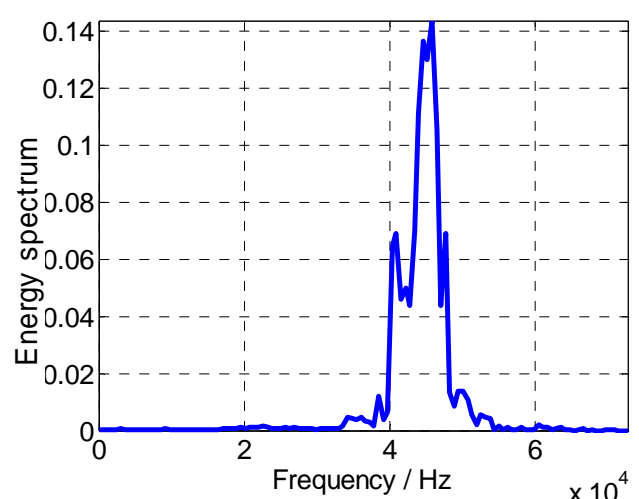

Figure 18. Energy Spectrum of Position with Hole

\section{CONCLUSION}

Experimental results show that the ultrasonic method combining with the filtering process and HHT is feasible to inspect the quality of CFST. No matter the defect is delamination between the steel and concrete, sundry inside the concrete or small hole inside the concrete, it can be effectively recognized. However, further investigation is needed to determine the size of the defect. And, for concrete filled hollow steel tube, it would be difficult to recognize the defect in it, efforts is needed to distinguish the effect of the hole in the concrete to the ultrasonic signal. 


\section{REFERENCES}

[1] Wu, X.X., "Concrete Nondestructive Testing Technology Manual”, China Communications Press, 1999 (in Chinese).

[2] Technical Specification for Ultrasonic Testing of Concrete Defects (CECS21:90), China Engineering Construction Standardization Association (in Chinese).

[3] Zhou, X.Y., Xiao, Y.F. and Cao, G.H., "Investigation into the Ultrasonic Method for the Quality of CFST, Railway Science and Engineering. 2006, Vol. 6, No. 3, pp. 50-54 (in Chinese).

[4] Xu, S.W. and Shi, H., "First Exploration of Time-Frequency Analysis Method", Modern Electronics, 1996, Vol. 54, No. 1, pp. 38-44 (in Chinese).

[5] Braun, S. and Feldman, M., "Time-frequency Characteristics of Non-linear Systems", Mechanical Systems and Signal Processing, 1997, Vol. 11, No. 4, pp. 611-620.

[6] Norden, E., Huang, Z.S., Steven, R. and Long, et al., "The Empirical Mode Decomposition and the Hilbert Spectrum for Nonlinear and Non-stationary Time Series Analysis", Proc. R. Soc. Lond. A., 1998, Vol. 454, pp. 899-955.

[7] Vesselin, V., "The Analysis of the Empirical Mode Decomposition Method", USC, November 20, 2002.

[8] Gong, M.S. and Xie, L.L., "Preliminary Study of the Application of HHT Method in Earthquake Engineering", World Earthquake Engineering, 2003, Vol. 19, No. 3 (in Chinese). 